\title{
Short Communication \\ Methylmercury induces the opening of the permeability transition pore in rat liver mitochondria
}

\author{
Marcantonio Bragadin $^{\mathrm{a}, *}$, Daniele Marton ${ }^{\mathrm{b}}$, Sabrina Manente ${ }^{\mathrm{a}}$, Mario Grasso ${ }^{\mathrm{a}}$, Antonio Toninello ${ }^{\mathrm{c}}$ \\ ${ }^{\mathrm{a}}$ Dipartimento di Scienze Ambientali, Università di Venezia, DD2137 30123 Venice, Italy \\ ${ }^{\mathrm{b}}$ Dipartimento di Chimica Inorganica, Metallorganica e Analitica, Università di Padova, Via Marzolo 1, 35131 Padua, Italy \\ ${ }^{\mathrm{c}}$ Dipartimento di Chimica Biologica e centro Studio delle Biomembrane del C.N.R., Università di Padova, Viale G. Colombo 3, 35131 Padua, Italy
}

Received 21 March 2001; received in revised form 28 August 2001; accepted 19 September 2001

\begin{abstract}
Interactions of methylmercury $\left(\mathrm{CH}_{3} \mathrm{HgCl}\right)$ with non-energized mitochondria from rat liver (non-respiring mitochondria) have been investigated in this paper. It has been shown that $\mathrm{CH}_{3} \mathrm{HgCl}$ induces swelling in mitochondria suspended in a sucrose medium. Swelling has also been induced by detergent compounds and by phenylarsine, a chemical compound which induces opening of the permeant transition pore (MTP). Opening of the MTP is inhibited by means of cyclosporine A. Results indicate that the swelling induced by $\mathrm{CH}_{3} \mathrm{HgCl}$, as in the case of phenylarsine, is inhibited by cyclosporine $\mathrm{A}$ and $\mathrm{Mg}^{2+}$, while swelling induced by detergent compounds is not cyclosporine sensitive. This comparison suggests that $\mathrm{CH}_{3} \mathrm{HgCl}$ induces opening of a permeability transition pore (MTP). Since the opening of an MTP induces cell death, this interaction with MTP could be one of the causes of toxicity of $\mathrm{CH}_{3} \mathrm{HgCl}_{2}$ (C) $2002 \mathrm{Elsevier}^{-}$ Science Inc. All rights reserved.
\end{abstract}

Keywords: Methylmercury; Uncoupling; Mitochondria

Methylmercury $\left(\mathrm{CH}_{3} \mathrm{HgCl}\right)$ has been shown by many investigators to have toxic effects on animals, as well as on humans [1-6]. Although these studies have clearly identified the central nervous system as the primary target for the cytotoxic action of methylmercury, the reason for such selectivity on the part of this compound is presently unknown. Furthermore, the molecular mechanisms underlying the cytotoxic effects of methylmercury have not been completely clarified. In whole cells, an increase in the cytosolic $\mathrm{Ca}^{2+}$ concentration, as a consequence of treatment with methylmercury, was observed in rat cerebrum synaptosomes [7], in PC12 cells [8], and in rat T lymphocytes [9]. Inhibition of ATP synthesis as a consequence of destruction of the transmembrane potential and apoptosis in cellular systems have also been reported [10-13].

Many 'in vitro' studies suggest that in the cell, the mitochondria are the preferential target for methylmercury [14-19]. In this regard, the most significant effects are:

\footnotetext{
*Corresponding author. Tel.: +39-041-257-8511; fax: +39-041-2578584.

E-mail address: bragadin@unive.it (M. Bragadin).
}

- an increase in potassium permeability in the inner membrane [14-16],

- inhibition of the respiratory chain and collapse of the mitochondrial membrane potential [14-17],

- calcium release [16-18], and

- cytochrome c release [19].

In this paper we present evidence of an additional effect, since results from our study showed that methylmercury induces the opening of the permeability pore (MTP) in rat liver [20] non-respiring mitochondria. This opening allows for the passage of large molecules with molecular masses of $\sim 1500 \mathrm{Da}$ [22-25]. The presence and the activity of the MPT pore is evidenced by means of swelling experiments [22-25]. As the opening of the MTP channel is one of the causes of apoptosis, this could contribute to an explanation of the toxicity and of the previously noted ion leak in mitochondria.

The inner membrane of mitochondria cannot be permeated by many chemical compounds. In general, swelling, which is the consequence of the passage of solutes through the inner membrane, can be produced by three mechanisms: (i) detergent compounds induce enhancement of the membrane permeability, since their hydrophobic 
chain alters the physical state of the phospholipid bilayer; (ii) many chemical compounds induce the opening of an MTP of large size, which allows for the transport of large molecules with large molecular masses of $\sim 1500 \mathrm{Da}[22-$ 25] (the MTP opening is inhibited by the presence of cyclosporin A); and (iii) the activities of many uniporters and antiporters together with the transport of anions such as $\mathrm{NO}_{3}^{-}$induce swelling in mitochondria [22]. For example, in an acetate medium, deenergized mitochondria swell at a rapid rate if $\mathrm{Na}^{+}$is present in the medium. The swelling is due to the presence of an $\mathrm{Na}^{+} / \mathrm{H}^{+}$exchanger. Analogously, swelling occurs in energized mitochondria in a $\mathrm{K}^{+}$medium only in the presence of the potassium carrier, valinomycin [22]. Regarding all the mechanisms, the entry of a solute is accompanied by the entry of water and swelling occurs by means of a colloid osmotic mechanism [26].

Fig. 1a shows the swelling induced in mitochondria by sodiumdodecylsulphate (SDS), a detergent compound.

The swelling is not inhibited by the presence of cyclosporin $\mathrm{A}$, thus suggesting that it is due to a modification in the phospholipid bilayer (mechanism i). Fig. 1b shows the swelling induced in mitochondria by phenylarsine $(\mathrm{PhA})$, a potent inducer of the opening of the transition pore [27]. This swelling is inhibited by the presence of cyclosporin A since, in this case, it results from the opening of a cyclosporin A-sensitive transition pore [22-25] (mechanism ii). Fig. 1c shows the swelling induced in mitochondria by the methylmercury chloride $\mathrm{CH}_{3} \mathrm{HgCl}$. The rate of swelling (i.e. the slope of the absorbance change

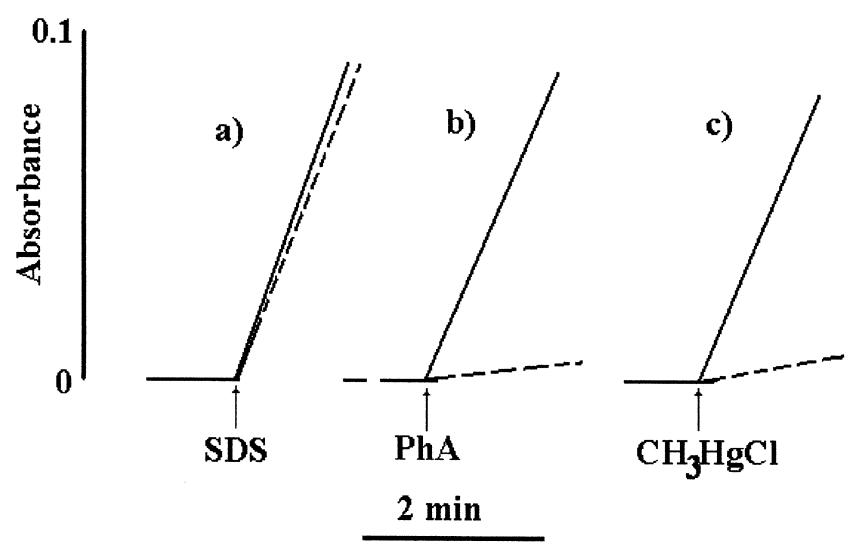

Fig. 1. Swelling induction in non-energized mitochondria. Composition of the medium: $0.25 \mathrm{M}$ sucrose, $10 \mathrm{mM}$ Tris-Hepes $\mathrm{pH} 7.4,1 \mu \mathrm{M}$ rotenone. Mitochondria [20] $(0.5 \mathrm{mg} / \mathrm{ml}$; the protein concentration was monitored using the Lowry procedure [21]) were added to $2.5 \mathrm{ml}$ medium. After the addition of the medium and the mitochondria, the spectrophotometer (Jenway 6400) was adjusted (at $540 \mathrm{~nm}$ ) at zero absorbance before adding the methylmercury. Since the swelling causes a decrease in absorbance, the absorbance change appears as negative value. The arrows indicate the addition (unbroken line) of the following compounds to the medium containing mitochondria: (a) $2.6 \mu \mathrm{M}$ sodiumdodecylsulphate (SDS), (b) $10 \mu \mathrm{M}$ phenylarsine (PhA), (c) $10 \mu \mathrm{M}$ $\mathrm{CH}_{3} \mathrm{HgCl}$ (Aldrich). In each case, the dashed line indicates the same experiment in the presence of $1 \mu \mathrm{M}$ cyclosporin A (SIGMA). against time) depends on the $\mathrm{CH}_{3} \mathrm{HgCl}$ concentration (not shown here) and is inhibited by the presence of cyclosporin A. A comparison between the mechanisms in Fig. 1 suggests that $\mathrm{CH}_{3} \mathrm{HgCl}$ induces swelling by the permeability transition pore mechanism because it is inhibited by the presence of cyclosporin A. In this regard it should be noted that cyclosporine $\mathrm{A}$ is the most efficient and selective inhibitor of MTP opening [22-25]. Therefore, the cyclosporine A response is the check-in test to demonstrate the presence of the MTP pore.

Other swelling mechanisms such as those indicated in (iii) are excluded, since solutes such as $\mathrm{K}^{+}, \mathrm{Na}^{+}$and $\mathrm{NO}_{3}^{-}$, are not present in the medium and none of these mechanisms, which depend on the presence of uni/antiporters and ions such as $\mathrm{K}^{+}, \mathrm{Na}^{+}, \mathrm{NO}_{3}^{-}$, are cyclosporinsensitive.

Further confirmation of the results arises from experiments regarding swelling in the presence of $\mathrm{Mg}^{2+}$ which inhibits and retards the opening of the MTP [22-25]. In this case the opening of the pore occurs in the presence of higher doses of $\mathrm{CH}_{3} \mathrm{HgCl}$ and is inhibited by the cyclosporin (Fig. 2). The swelling induced by $\mathrm{CH}_{3} \mathrm{HgCl}$ has already been observed [14] in mitochondria, but the experimental conditions and the interpretation of the data are different from those presented in this manuscript. In Ref. [14], the swelling was observed in a medium containing potassium: the interpretation presented was that in
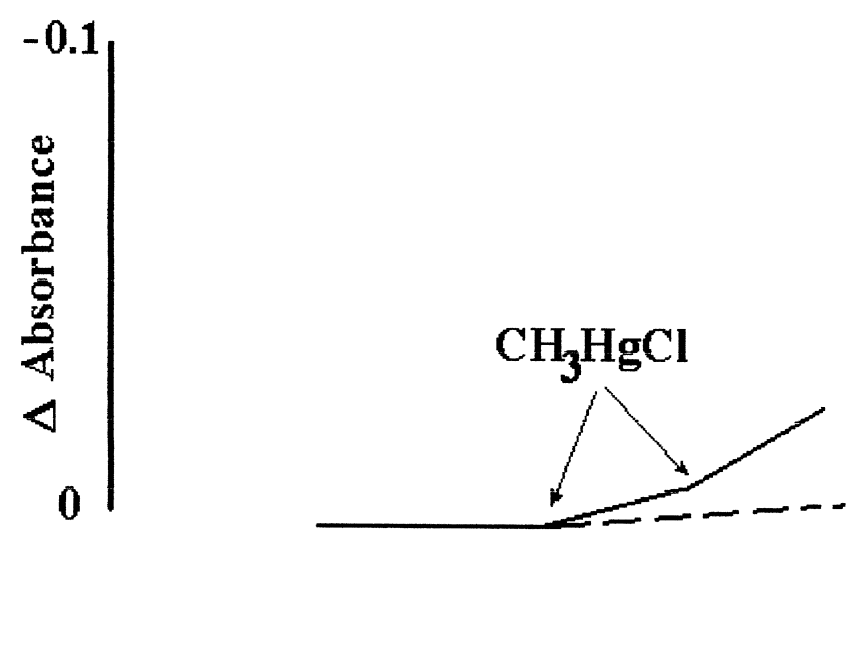

$2 \min$

Fig. 2. Swelling of mitochondria in a medium containing magnesium. Composition of the medium: $0.25 \mathrm{M}$ sucrose, $10 \mathrm{mM}$ Tris-Hepes $\mathrm{pH}$ 7.4, $1 \mu \mathrm{M}$ rotenone and $5 \mathrm{mM} \mathrm{MgCl}$. Mitochondria $(0.5 \mathrm{mg} / \mathrm{ml})$ were added to $2.5 \mathrm{ml}$ medium. Arrows indicate two successive additions of $20 \mu \mathrm{M}$ $\mathrm{CH}_{3} \mathrm{HgCl}$ to the medium containing the mitochondria. Dashed line: the same experiment in the presence of $1 \mu \mathrm{M}$ cyclosporin A. The reproducibility of the experiments was verified by means of four different mitochondrial preparations and the changes in the swelling rate (the rate of absorbance change against time) were no more than 5\%. The experiment in this figure and in Fig. 1 is a typical experiment. 
energized mitochondria $\mathrm{CH}_{3} \mathrm{HgCl}$ increases the potassium permeability and consequently allows for the uptake of potassium, thus inducing swelling. The potential quenching of the membrane has been found to be the consequence of this potassium uptake. Furthermore the effect produced by cyclosporin has not been considered.

In the experiments in Fig. 1c, the swelling induced by $\mathrm{CH}_{3} \mathrm{HgCl}$ was observed in a potassium free medium in non-energized mitochondria. Consequently, the most significant evidence to support the opening of the MTP is the fact that the swelling is inhibited by the presence of cyclosporin A. In the light of this behaviour, the experiments in Ref. 14 can be explained not as resulting from the influx of potassium, but rather from the influx of sucrose. The opening of the MTP results in the transport of protons through the membrane (uncoupling effect) and this could thus explain the collapse of the membrane potential; the previously observed $\mathrm{Ca}^{2+}$ release [16-18] is a consequence of this phenomenon.

With regard to mitochondria, many organometallic compounds such as alkyl ${ }_{3} \mathrm{SnCl}$ and alkyl ${ }_{3} \mathrm{PbCl}$ induce swelling [28-32]. In Refs. [28,30] the swelling has been interpreted as resulting from an electroneutral $\mathrm{OH}^{-} / \mathrm{Cl}^{-}$ exchange: the organometal compound enters the matrix as alkyl $_{3} \mathrm{SnCl}$ (or alkyl ${ }_{3} \mathrm{PbCl}$ ) and is extruded as alkyl ${ }_{3} \mathrm{SnOH}$ (or alkyl ${ }_{3} \mathrm{PbOH}$ ), thus giving rise to a cyclic mechanism, where the balance during any cycle results in an electroneutral $\mathrm{Cl}^{-} / \mathrm{OH}^{-}$exchange. This mechanism, which has been proposed in order to explain the toxicity of alkyl ${ }_{3} \mathrm{SnCl}$ and alkyl $_{3} \mathrm{PbCl}$, cannot explain the results of the experiments in Fig. 1c, since $\mathrm{Cl}^{-}$is absent from the medium. Furthermore, a single uptake and accumulation of $\mathrm{CH}_{3} \mathrm{HgCl}$ or $\mathrm{CH}_{3} \mathrm{HgOH}$ cannot be proposed as a swelling promoter because of the low $\mathrm{CH}_{3} \mathrm{HgCl}$ concentration. In any case, this mechanism would not be cyclosporine Asensitive.

It must be noted that, in our conditions, i.e. with regard to non-respiring mitochondria (in the absence of reducing substrates and consequently in non-energized conditions), it is possible to focus attention only on one aspect of the interaction between methylmercury and mitochondria (the swelling), while in energized conditions, the inhibitory effect on the respiratory chain [16] and the interactions with the ion permeability in the membrane overlap [14$16]$.

In conclusion, the mechanism proposed in this paper, i.e. the opening of a pore which allows for the transport of large molecules, can explain the most significant phenomena already observed in mitochondria:

- the release of $\mathrm{Ca}^{2+}$ and the transport of $\mathrm{K}^{+}$,

- the uncoupling effect and collapse of the potential, since the opening of the pore enhances the membrane permeability to all ions, the protons enclosed (uncoupling effect), and
- the release of cytochrome c.

In this regard, the cytochrome c release occurs when the mitochondria undergoes swelling [33]. Therefore, this effect could be an indirect consequence of the induction of MTP. The apoptosis and cell death can easily be explained, since the opening of the cyclosporin A-sensitive pore is one of the causes of apoptosis.

\section{Abbreviations}

\begin{tabular}{|c|c|}
\hline $\mathrm{Da}$ & Dalton \\
\hline Hepes & $\begin{array}{l}\left(N \text {-[2-Hydroxyethyl]piperazine- } N^{\prime}-\right. \\
\text { [2-ethanesulphonic acid]) (Sigma, }\end{array}$ \\
\hline MTP & Permeability transition pore \\
\hline $\mathrm{PhA}$ & Phenylarsine oxide (Aldrich, Milan) \\
\hline SDS & Sodiumdodecylsulphate (Sigma, Milan) \\
\hline Tris & $\begin{array}{l}\text { 2-Amino-2-(hydroxymethyl)-1,3-propanediol } \\
\text { (Sigma, Milan) }\end{array}$ \\
\hline
\end{tabular}

\section{Acknowledgements}

We are grateful to Michele Gallo for his technical assistance.

\section{References}

[1] T. Takeuchi, N. Norikawa, H. Matsumoto, Y. Shiraishi, Acta Neuropathol. 2 (1962) 40.

[2] F. Bakir, S.F. Damluji, L. Amin-Zaki, M. Murtadha, A. Khalidi, N.Y. Al-Rawi, S. Tikriti, H.I. Dahair, T.W. Clarkson, J.C. Smith, R.A. Doherty, Science 182 (1973) 230.

[3] C.R. Adams, D.K. Ziegler, J.T. Lin, J. Am. Med. Assoc. 250 (1983) 642.

[4] J.C. Hansen, in: A.W. Fumess, P.S. Rainbow (Eds.), Heavy Metals in the Marine Environment, CRC Press, New York, 1990, pp. $227-$ 243.

[5] L.W. Chang, in: P.S. Spencer, H.H. Schauenburg (Eds.), Experimental and Clinical Neurotoxicology, Williams and Williams, New York, 1980, pp. 508-526.

[6] L.W. Chang, Environ. Res. 14 (1977) 329.

[7] H. Komulainen, S.C. Bondy, Toxicol. Appl. Pharmacol. 88 (1987) 77.

[8] M.F. Hare, W.D. Atchinson, Toxicologist 12 (1992) 313.

[9] T. Xiao Xia, T. Cuye, A. Castoldi, L. Manzo, L. Costa, J. Toxicol. Environ. Health 38 (1993) 159.

[10] A.H. Close, T.L. Guo, B.J. Shenker, Toxicol. Sci. 49 (1999) 68.

[11] B.J. Shenker, T.L. Guo, J.M. Shapiro, Environ. Res. 77 (1998) 149.

[12] J.D. Robertson, J.D. Orrenius, Crit. Rev. Toxicol. 30 (2000) 609.

[13] B.J. Shenker, T.L. Guo, I.M. Shapiro, Environ. Res. 84 (2000) 89.

[14] N. Sone, M. Larsstuvold, Y. Kagawa, J. Biochem. 82 (1977) 859.

[15] M.A. Verity, W. Brown, M. Cheung, J. Neurochem. 25 (1975) 759.

[16] W. Atchinson, M. Hare, FASEB J. 8 (1994) 622.

[17] S.C. Bondy, M. McKee, Toxicol. Lett. 58 (1991) 13.

[18] M.F. Denny, M.F. Hare, W.D. Atchison, Toxicol. Appl. Pharmacol. 122 (1993) 222.

[19] B.J. Shenker, T.L. Guo, J.M. Shapiro, Toxicol. Appl. Pharmacol. 157 (1999) 23. 
[20] S. Massari, E. Balboni, G.F. Azzone, Biochim. Biophys. Acta 283 (1972) 16.

[21] O.H. Lowry, N.J. Rosenbrough, A.L. Farr, R.J. Randall, J. Biol. Chem. 193 (1951) 265.

[22] P. Bernardi, Physiol. Rev. 79 (1999) 1127.

[23] P. Bernardi, L. Scorrano, R. Colonna, V. Petronilli, F. Di Lisa, Eur. J. Biochem. 264 (1999) 687.

[24] V. Petronilli, D. Penzo, L. Scorrano, P. Bernardi, F. Di Lisa, J. Biol. Chem. 276 (2001) 12030.

[25] P. Bernardi, V. Petronilli, J. Bioenerg. Biomembr. 28 (1996) 129.

[26] G.F. Azzone, A. Azzi, Proc. Natl. Acad. Sci. USA 53 (1965) 1084.
[27] M. Zoratti, I. Szabò, Biochim. Biophys. Acta 1241 (1995) 139.

[28] D.N. Skilleter, Biochem. J. 146 (1975) 465.

[29] M. Bragadin, D. Marton, M. Murgia, V. Rizzoli, G. Scutari, R. Deana, J. Inorg. Biochem. 69 (1998) 259.

[30] W.N. Aldridge, B.W. Street, D.N. Skilleter, Biochem. J. 168 (1977) 353.

[31] M. Bragadin, D. Marton, A. Toninello, E.R. Viola, Inorg. Chem. Commun. 3 (2000) 255.

[32] M. Bragadin, D. Marton, J. Inorg. Biochem. 68 (1997) 75.

[33] E.E. Jacobs, D.R. Sanadi, J. Biol. Chem. 235 (1960) 531. 